\title{
A Brief Review of Current Maturation Methods for Human Induced Pluripotent Stem Cells-Derived Cardiomyocytes
}

\author{
Razan Elfadil Ahmed ${ }^{1}$, Tatsuya Anzai 1,2, Nawin Chanthra ${ }^{1}$ and Hideki Uosaki ${ }^{1 *}$ \\ ${ }^{1}$ Division of Regenerative Medicine, Center for Molecular Medicine, Jichi Medical University, Shimotsuke, Japan, \\ ${ }^{2}$ Department of Pediatrics, Jichi Medical University, Shimotsuke, Japan
}

OPEN ACCESS

Edited by:

Jong-Kook Lee,

Osaka University, Japan

Reviewed by:

Katriina Aalto-Setälä,

University of Tampere, Finland

Thomas Owen,

Imperial College London, United Kingdom

*Correspondence:

Hideki Uosak

uosaki.hideki@jichi.ac.jp

Specialty section:

This article was submitted to

Stem Cell Research,

a section of the journal

Frontiers in Cell and Developmental

Biology

Received: 13 January 2020

Accepted: 03 March 2020

Published: 19 March 2020

Citation:

Ahmed RE, Anzai T, Chanthra N and Uosaki $H$ (2020) A Brief Review of Current Maturation Methods for Human Induced Pluripotent Stem

Cells-Derived Cardiomyocytes.

Front. Cell Dev. Biol. 8:178.

doi: 10.3389/fcell.2020.00178
Cardiovascular diseases are the leading cause of death worldwide. Therefore, the discovery of induced pluripotent stem cells (iPSCs) and the subsequent generation of human induced pluripotent stem cell-derived cardiomyocytes (hiPSC-CMs) was a pivotal point in regenerative medicine and cardiovascular research. They constituted an appealing tool for replacing dead and dysfunctional cardiac tissue, screening cardiac drugs and toxins, and studying inherited cardiac diseases. The problem is that these cells remain largely immature, and in order to utilize them, they must reach a functional degree of maturity. To attempt to mimic in vivo environment, various methods including prolonging culture time, co-culture and modulations of chemical, electrical, mechanical culture conditions have been tried. In addition to that, changing the topology of the culture made huge progress with the introduction of the 3D culture that closely resembles the in vivo cardiac topology and overcomes many of the limitations of the conventionally used 2D models. Nonetheless, 3D culture alone is not enough, and using a combination of these methods is being explored. In this review, we summarize the main differences between immature, fetal-like hiPSC-CMs and adult cardiomyocytes, then glance at the current approaches used to promote hiPSC-CMs maturation. In the second part, we focus on the evolving 3D culture model - it's structure, the effect on hiPSC-CMs maturation, incorporation with different maturation methods, limitations and future prospects.

Keywords: induced pluripotent stem cells, human induced pluripotent stem cells-derived cardiomyocytes, regenerative medicine, 3-dimensional culture, engineered heart tissue

\section{INTRODUCTION}

The generation of induced pluripotent stem cells (iPSCs) forever changed the field of regenerative medicine, basic and translational biomedical researches (Takahashi et al., 2007). Human iPSCs became an appealing alternative to embryonic stem cells (Bilic and Izpisua Belmonte, 2012; Puri and Nagy, 2012). Since Yamanaka's breakthrough, many efficient protocols have been developed for generating cardiomyocytes derived from human iPSCs (hiPSC-CMs) (Yang et al., 2008; Elliott et al., 2011; Uosaki et al., 2011; Burridge et al., 2012, 2014; Minami et al., 2012; Dunn and Palecek, 2018).

One of the unresolved problems is that hiPSC-CMs remain largely immature when compared to human adult cardiomyocytes. Such immaturity hinders their usage on many aspects, e.g., pharmacological and toxicological screening (Sinnecker et al., 2014) and cardiovascular disease 
modeling (Jung and Bernstein, 2014; Yang et al., 2015). Transplanting human embryonic stem cell-derived cardiomyocytes (hESC-CMs) or non-human primate iPSC-CMs into non-human primates model of myocardial ischemiareperfusion resulted in substantial remuscularization, but non-fatal ventricular arrhythmias were observed (Chong et al., 2014; Shiba et al., 2016), which could be a result of transplanting immature PSC-CMs that have automaticity. To address the issue of immaturity, many different approaches have been examined on enhancing the maturation of hiPSC-CMs.

In this review, we briefly list the main differences between immature, fetal-like hiPSC-CMs and adult cardiomyocytes (Figure 1). Then, we discuss the current methods used to promote hiPSC-CMs maturation (Figure 2). In the second part, we describe the details of the evolving $3 \mathrm{D}$ culture model - it's structure, the effect on hiPSC-CMs maturation, incorporation with different maturation methods, limitations, and future perspectives.

\section{COMPARISON OF hiPSC-CMs AND ADULT CARDIOMYOCYTES TO EVALUATE MATURITY}

Researchers in the stem cell field agree that hiPSC-CMs are immature, but there is no consensus about how to evaluate their degree of maturation. Therefore, in addition to developing ways to increase iPSC-CMs maturity, methods on how to assess maturation are required. Here, we summarize characteristics of adult cardiomyocytes and differences to hiPSCCMs (Figure 1).

\section{Morphology and Structure}

It takes up to 10 years for cardiomyocytes to acquire adult phenotypes in structure and ploidy in a human heart (Peters et al., 1994; Bergmann et al., 2009; Vreeker et al., 2014). Adult cardiomyocytes are well-aligned, rodlike, multinucleated/tetraploid cells, with highly organized sarcomeres, well developed sarcoplasmic reticulum (SR) and transverse tubules (T-tubules) (Peters et al., 1994; Bergmann et al., 2009; Yang et al., 2014a), and have intercalated disks with mature mechanical and electrical junctions (Dhamoon and Jalife, 2005; Zwi et al., 2009; Ma et al., 2011; Kamakura et al., 2013; Vreeker et al., 2014; Denning et al., 2016). Such phenotypical maturation is still lacking in hiPSCCMs, which tend to be small, mononucleated, rounded cells with disorganized sarcomere. Moreover, they have shorter sarcomeres, poorly developed SR, and no T-tubules (Yang et al., 2014a; Denning et al., 2016). Structural features can be used to evaluate the degree of hiPSC-CMs maturity because some of these features are characteristic to mature cardiomyocytes.

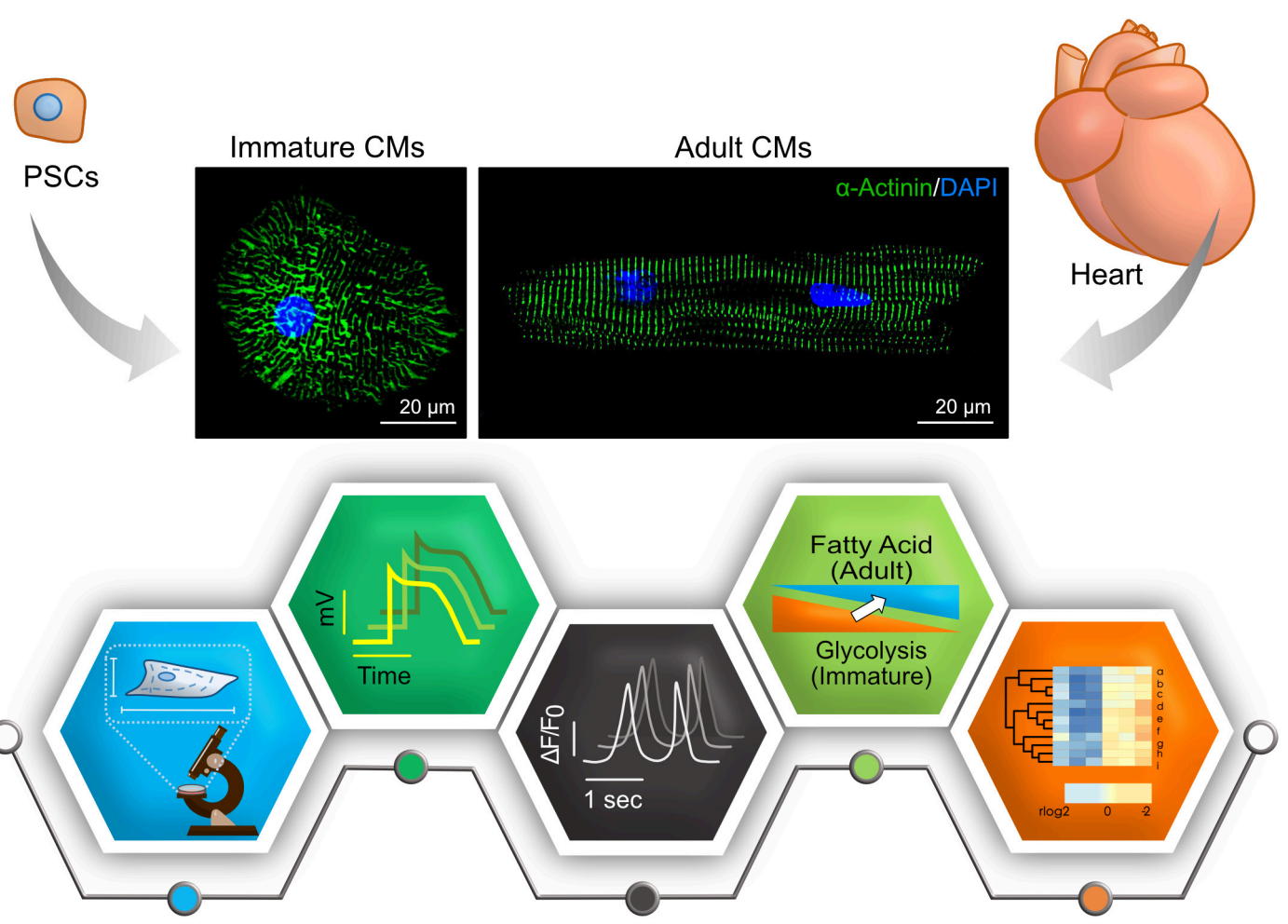

FIGURE 1 | Key features to characterize cardiomyocyte maturity. Compared to adult cardiomyocytes (CMs), many aspects - morphology and structure, electrophysiology, calcium handling, metabolism, and transcriptome - are different in immature PSC-CMs. Representative immunostainings of cardiomyocytes derived PSCs and adult mouse hearts are shown in upper panels (Green, $\alpha$-actinin; Blue, DAPI). Pictograms shown in lower panels represent each aspect. 


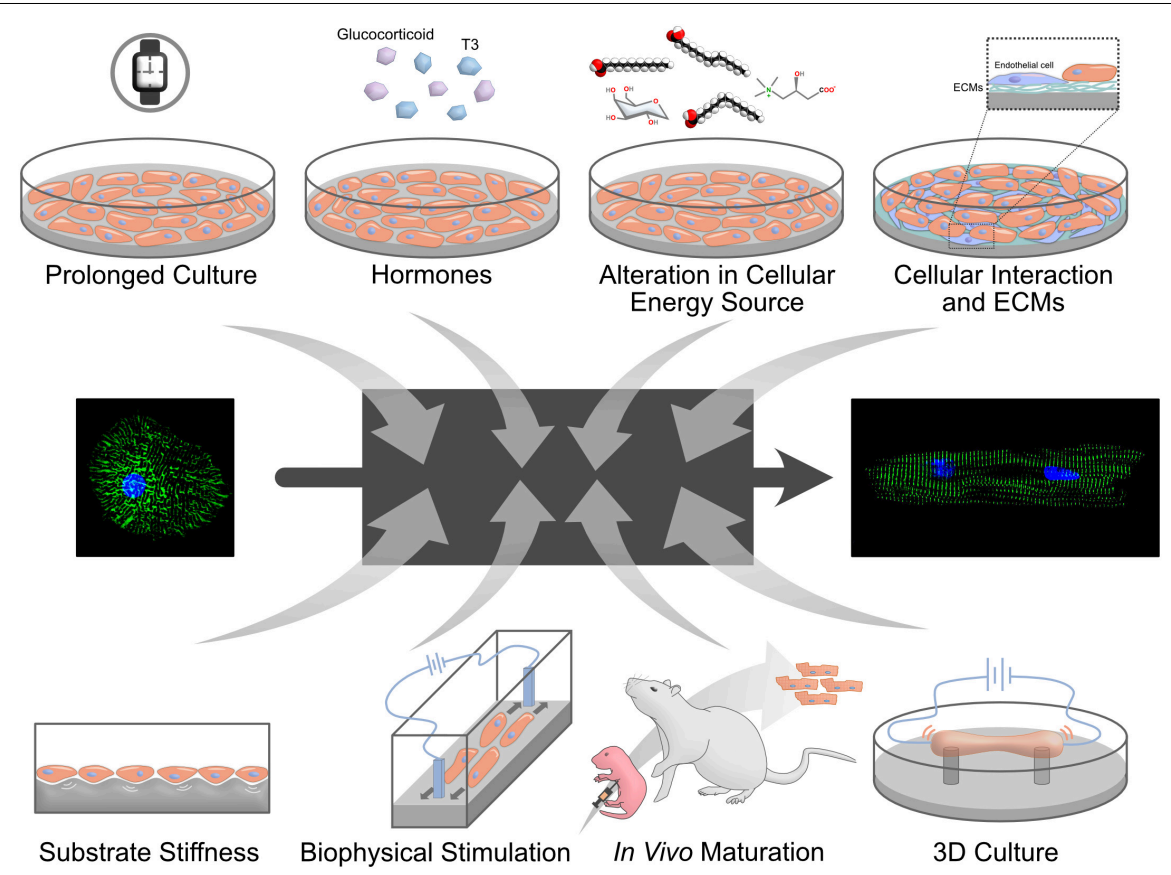

FIGURE 2 | Methods to mature PSC-CMs. Many different approaches to mature PSC-CMs have been proposed. However, it is largely unknown why these signals control cardiomyocyte maturation and how mature PSC-CMs can achieve as a result. A black box shown in the middle represents the unelucidated mechanisms.

\section{Physical and Electrophysiological Properties}

Adult cardiomyocytes only beat when stimulated with a force around $40-80 \mathrm{mN} / \mathrm{mm}^{2}$, conduction velocity around $60 \mathrm{~cm} / \mathrm{s}$ and upstroke velocity about $150-350 \mathrm{~V} / \mathrm{s}$. In hiPSC-CMs, these parameters are around $0.08-4 \mathrm{mN} / \mathrm{mm}^{2}, 10-20 \mathrm{~cm} / \mathrm{s}$, and $10-$ $50 \mathrm{~V} / \mathrm{s}$, consecutively (Denning et al., 2016). Moreover, hiPSCCMs display mixed action potential (AP) morphologies that can be categorized as atrial, nodal, or ventricular-like AP (Ma et al., 2011). Although hiPSC-CMs generate important cardiac currents such as $\mathrm{I}_{\mathrm{Na}}, \mathrm{I}_{\mathrm{Ca}, \mathrm{L}} \mathrm{I}_{\mathrm{to}}$, $\mathrm{I}_{\mathrm{Kr}}$, and $\mathrm{I}_{\mathrm{Ks}}$, they lack $\mathrm{I}_{\mathrm{K} 1}$ that is essential for stabilization of the resting potential (Dhamoon and Jalife, 2005; Hoekstra et al., 2012; Knollmann, 2013). This deficiency might be particularly important when hiPSC-CMs are used to study long QT syndrome. Human ether-a-go-go related gene (hERG) encode a subunit of $\mathrm{I}_{\mathrm{Kr}}$ channel, and mutation in hERG or blockade of $\mathrm{I}_{\mathrm{Kr}}$ cause long QT syndrome. Without $\mathrm{I}_{\mathrm{K} 1}$, hiPSCCMs rely on $\mathrm{I}_{\mathrm{Kr}}$ for the maximum diastolic potential (MDP) that is markedly depolarized with $\mathrm{I}_{\mathrm{Kr}}$ blockers (Doss et al., 2012). The other characteristic is the spontaneous beatings of hiPSC-CMs. $\mathrm{I}_{\mathrm{f}}$ current generated by HCN4, which is restricted to pacemaker cells in vivo, depolarize MDP and make hiPSC-CMs beat (Yanagi et al., 2007). Measuring the electrophysiological parameters and assessing the generation of $\mathrm{I}_{\mathrm{K} 1}$ current is a promising tool that can be utilized to assess the maturation of hiPSC-CMs, though it would be technically challenging.

\section{Calcium Handling}

In adult cardiomyocytes, T-tubules and SR are well developed to regulate $\mathrm{Ca}^{2+}$ induced $\mathrm{Ca}$ release (CICR) and fast excitation-contraction coupling (ECC). The inflow of $\mathrm{Ca}^{2+}$ via L-type channels triggers the release of $\mathrm{Ca}^{2+}$ from the SR through the ryanodine receptor (RyR) channels (Bers, 2002). T-tubules, invagination of the cell membrane, near L-type $\mathrm{Ca}^{2+}$ channel and RyR in adult cardiomyocytes. In the relaxation phase, $\mathrm{Ca}^{2+}$ is returned to the SR through sarco/endoplasmic reticulum $\mathrm{Ca}^{2+}$ ATPase (SERCA) and is extruded from the cell through the $\mathrm{Na}^{+}-\mathrm{Ca}^{2+}$ exchanger (NCX). The sharp and uniform increase of intracellular $\mathrm{Ca}^{2+}$ concentration in adult cardiomyocytes is important for the synchronized contraction in multiple sarcomeres (Scuderi and Butcher, 2017; Steinhoff et al., 2017). In hiPSC-CMs, T-tubules are absent and SR is underdeveloped with low expression of SERCA and other key proteins. As a result, hiPSC-CMs rely on L-type channels for the increase of $\mathrm{Ca}^{2+}$ and ECC is slow (Pesl et al., 2017; Veerman et al., 2017).

\section{Metabolism}

In adult cardiomyocytes, mitochondria volume increases, and the oxidative capacity is increased, which represents to switch in metabolic substrates from glucose to fatty acid (Lopaschuk and Jaswal, 2010). To examine glycolysis and fatty acid oxidation, the oxygen consumption rate (OCR) and the extracellular acidification rate (ECAR) are often used, respectively (Rana et al., 2012). During early heart development, around $80 \%$ of energy is produced by glycolysis. When cardiomyocytes become mature, fatty acid $\beta$-oxidation increases and becomes a major source for energy production. In a rabbit, the metabolic switch occurs during early postnatal growth (Lopaschuk et al., 1991). As hiPSC$\mathrm{CMs}$ remain immature, they rely on glycolysis rather than fatty 
acid $\beta$-oxidation (Rana et al., 2012; Kim et al., 2013; Kikuchi et al., 2015; Correia et al., 2017).

\section{Gene Expression}

Identifying the genes involved in human cardiomyocytes maturation is still an ongoing process. But the overall expression pattern of maturation-related genes identified in mice and humans are mostly similar (DeLaughter et al., 2016; Uosaki and Taguchi, 2016). Isoform transitions of sarcomeric genes occur from fetal to adult period. Cardiac myosin heavy chain (MHC, also known as $M Y H$ ) has two isoforms. These are $\alpha$-isoform $(\alpha-M H C$, also known as MYH6) and $\beta$-isoform ( $\beta-M H C$, also known as MYH7). In adult cardiomyocytes, the $\beta$-isoform is predominant. To note, the isoform switch occurs from $\beta$-isoform to $\alpha$-isoform in mouse hearts. Troponin I (TnI) has three isoforms [slow skeletal (ssTnI), fast skeletal (fsTnI), and cardiac (cTnI)] encoded by TNNI1, TNNI2, and TNNI3, respectively. In adult cardiomyocytes, $\mathrm{cTnI}$ is highly expressed although ssTnI is the primary isoform in hiPSC-CMs. Titin (TTN) has three major isoforms. These are N2B, N2BA and fetal cardiac titin (FCT). In adult cardiomyocytes, N2B is mainly expressed whereas N2BA is predominant in hiPSC-CMs (Yin et al., 2015; Denning et al., 2016). Moreover, hiPSC-CMs show low expression levels of important cardiac genes such as SERCA2 (sarcoplasmic reticulum ATPase), CAV3 (caveolin 3), KCNH2 (potassium voltage-gated channel), and other adult cardiomyocytes genes (Karakikes et al., 2015; van den Berg et al., 2015; Denning et al., 2016).

To assess hiPSC-CMs maturation, measuring the TNNI3 to TNNI1 ratio is one way (Bedada et al., 2014). To achieve more precise measurement of maturation, transcriptomebased approaches were proposed, including a gene regulatory network-based (Uosaki et al., 2015) and a relative expression orderings-based scoring method (Chen et al., 2019). Singlecell transcriptome analysis could also predict the developmental ages of cardiomyocytes. However, most of these approaches are limited to mouse PSC-CMs as limited transcriptome data is available for full-spectrum of human developing hearts including late fetal and early postnatal periods (van den Berg et al., 2015; Kreipke et al., 2016; Tiburcy et al., 2017; Cardoso-Moreira et al., 2019). Thus, further work must be done to generate a valid, agreed upon, maturation index for hiPSC-CMs.

\section{CUES TO PROMOTE MATURATION OF hiPSC-CMs}

In order to be able to fully utilize hiPSC-CMs for clinical or research purposes, especially for drug discoveries and disease modeling, they must acquire an adult-like maturation state. In this part, we discuss different approaches used to enhance the maturation of hiPSC-CMs (Figure 2).

\section{Prolonged Culture Time}

It takes years for cardiomyocytes to fully mature in vivo (Vreeker et al., 2014), which prompted a hypothesis that prolonged culture time would promote maturation of hiPSC-CMs. To date, hiPSCCMs were cultured up to a full year to test their maturity
(Kamakura et al., 2013; Lundy et al., 2013; Lewandowski et al., 2018). With prolonged culture, hiPSC-CMs displayed more mature phenotypes in morphology (larger cell size), structure (myofibril density, alignment, microscopically visible sarcomere), and physiology (calcium handling and $\beta$-adrenergic response). The cells expressed maturation-related cardiac genes such as MYH7 with isoform switch (Lundy et al., 2013; Lewandowski et al., 2018). Interestingly, extending the cultures to 180 days resulted in more tightly packed myofibrils with the appearance of mature Z-, A-, H-, and I-bands, but not M-bands. M-bands, a key feature of sarcomere structure, are finally developed after 360 days of culture (Kamakura et al., 2013). These results consolidate the fact that prolonged culture generates more mature cardiomyocytes. However, it poses a question if it is possible to yield cells mature enough in a realistic, and financially appropriate culture time frame.

\section{Biochemical Cues Hormones}

Thyroid hormone, known to have a crucial role in cardiac development and cardiovascular physiology (Klein and Ojamaa, 2001), displayed strong enhancement of hiPSC-CM maturation (Yang et al., 2014b). Triiodothyronine (T3) treatment makes hiPSC-CMs bigger, more elongated morphology with longer sarcomeres. T3-treated hiPSC-CMs displayed increased mitochondrial activity and improved calcium handling along with higher contractile force.

Glucocorticoids are essential for maturation of fetal heart structure and function. Endogeneous glucocorticoids work by stimulating glucocorticoid receptor (GR) on fetal cardiomyocytes/vascular smooth muscle to promote myofibril assembly and organization (Rog-Zielinska et al., 2013, 2015). Adding glucocorticoid analog, dexamethasone, to T3 in culture further improve hiPSC-CMs maturation (Parikh et al., 2017).

These results highlight the importance of these chemical cues and call for studying more molecules and combinations that may further enhance maturation of cardiomyocytes.

\section{Alterations in Cellular Energy Source}

A hallmark of postnatal cardiomyocyte maturation is switching their metabolism from glycolysis to fatty acid oxidation (Yang et al., 2019). Recently, the glucose-free and lactate-containing medium were identified to eliminate non-cardiomyocyte and enrich hiPSC-CMs (Tohyama et al., 2013; Burridge et al., 2014), while hiPSC-CMs are usually cultured in glucose-containing medium. Replacing glucose with galactose and fatty acids - more specifically, palmitate, oleic acid, linoleic acid, and carnitine enhanced maturation of hiPSC-CMs (Correia et al., 2017; Nakano et al., 2017; Horikoshi et al., 2019; Yang et al., 2019). The switch of energy source not only increased mitochondrial number and metabolisms but also enhanced morphological, structural and physiological maturation. On the other hand, culturing hiPSC-CMs in high glucose medium inhibits their structural and functional maturation by promoting nucleotide biosynthesis (Nakano et al., 2017), which is attributed to a reduction of cardiac glucose uptake and increased nucleotide deprivation during late gestational and early postnatal stages. 


\section{Cellular Interaction and Extracellular Matrices}

Cells interact with each other through direct cellular contact or indirect paracrine factors secreted by the neighboring cells in a heart, and cellular interaction has been implicated in cardiac maturation (Talman and Kivelä, 2018; Yoshida et al., 2018; Abecasis et al., 2019). To mimic these cellular interactions in vitro, hiPSC-CMs were cocultured with non-cardiomyocytes, such as human mesenchymal stem cells (MSCs) and endothelial cells. Human MSCs secrete VEGF, bFGF, SDF-1, and GM-CSF to mediate differentiation and electrical coupling of hiPSC-CMs (Yoshida et al., 2018). In contrast, endothelial cells express extracellular matrices (ECMs; collagens I and III, fibronectin, thrombospondin-4) to increase sarcomere length of hiPSC-CMs (Abecasis et al., 2019). ECMs alone had some effects on enhancing structural and functional maturation of hiPSC-CMs (Chun et al., 2015; Herron et al., 2016; Ogasawara et al., 2017).

\section{Substrate Stiffness}

Extracellular matrices regulate tissue stiffness, and the stiffness of a heart increases gradually in vivo as a result of collagen accumulation (Jacot et al., 2010). This process increases the ability of the heart to pump blood due to its increased stiffness. Compared to hearts $(\sim 10 \mathrm{kPa})$, cell culture dishes are much stiffer $(\sim 1 \mathrm{MPa})$, which prompted a hypothesis that soft matrices might be better for the maturation of hiPSC-CMs. Polydimethylsiloxane (PDMS), hydrogels or polyacrylamide (PAA) were often used to create such culture conditions. Soft surfaces $(6 \sim 10 \mathrm{kPa})$ tuned sarcomere tension and contractility, and hiPSC-CMs on it generated more force $(0.1 \mu \mathrm{N})$ than those on a hard surface (35 kPa, 0.01-2 $\mu \mathrm{N}$ ) (Ribeiro et al., 2015). PDMS and PAA are also used to regulate topology of cell morphology and forcing hiPSC-CMs into rectangular shape results in better maturation (Thavandiran et al., 2013; McCain et al., 2014; Ribeiro et al., 2015). In the agreement with this, the vascular structure also forces hiPSC-CMs in a rectangular shape and made them more mature (Vuorenpää et al., 2017).

\section{Biophysical Stimulation}

Biophysical stimulation is absent under a standard culture condition. In a heart, cardiomyocytes are regularly exposed to electrical stimulation and mechanical stress. Applying a continuous electrical stimulation yielded hiPSC-CMs with rod-like morphology, enhanced cellular alignment, and more organized sarcomeres (Chan et al., 2013). Furthermore, subjecting combined synchronized electrical and mechanical stimulation on hiPSC-CM resulted in enhanced localization of $\mathrm{N}$-cadherin toward cell membrane, sarcomere shortening, and reduced transmembrane calcium current, suggesting more mature phenotype (Kroll et al., 2017).

\section{In vivo Maturation}

Instead of mimicking in vivo environment in vitro, an in vivo environment can be used to provide all necessary signals including unknown ones to hiPSC-CMs. There are some disagreements what developmental stages of the heart can be appropriate - neonate, adult, or adult heart after infarction (Funakoshi et al., 2016; Cho et al., 2017; Kadota et al., 2017), however, hiPSC-CMs in vivo are more matured than ones in vitro. Transplantation to neonatal hearts generated adultlike mature hiPSC-CMs within 2 months of transplantation, suggesting that the maturation speed is accelerated in a rat heart and it is defined by non-cell autonomous manner. Furthermore, hiPSC-CMs from a patient of arrhythmogenic right ventricular dysplasia/cardiomyopathy recapitulated disease phenotypes with the neonatal transplantation method (Cho et al., 2017).

\section{D CULTURE}

Cells are aligned three-dimensionally in vivo rather than a monolayer. In the last decade, 3D culture methods have been advanced and become an appealing alternative to the conventional 2D monolayer culture for the maturation of hiPSCCMs. As 3D tissues also resemble native cardiac architecture, 3D tissues of dilated cardiomyopathy hiPSC-CMs could recapitulate disease phenotypes that $2 \mathrm{D}$ cultures failed (Hinson et al., 2015). Here, we discuss the usefulness of $3 D$ cultures on the maturation of hiPSC-CMs.

\section{General Concepts of 3D Culture}

Conventional 2D cultures fail to recapitulate the complexity of the in vivo cellular crosstalk, tissue architectures, and extracellular microenvironments with forced and unwanted apical-basal polarity. On the other hand, 3D cultures have more similarity to the in vivo extracellular microenvironment, support better cellular interactions, and allows for biochemical and physical stimuli to reach the cells in an evenly distributed manner (Duval et al., 2017; Mirbagheri et al., 2019). In 3D cultures, hiPSCCMs display structural, functional and metabolic maturation compared to that in 2D cultures (Huethorst et al., 2016; Lemoine et al., 2017; Correia et al., 2018; Ulmer et al., 2018). They show improved myofibrillar alignment and sarcolemma remodeling which led to better $\mathrm{Ca}^{2+}$ handling (Silbernagel et al., 2020). Moreover, hiPSC-CMs in 3D culture exhibited faster maturation analyzed by transcriptome (Branco et al., 2019), while 2D cultures hinder the maturation or cause maturation arrest (Uosaki et al., 2015).

Different strategies are used to produce 3D cardiac tissues. One way is seeding hiPSC-CMs in to designed scaffolds or embedding them in hydrogel (Lemoine et al., 2017; Correia et al., 2018; Dattola et al., 2019; Silbernagel et al., 2020). To produce scaffold, photolithography is often used, however, it requires clean room and specialized equipment that are not often available for biomedical research labs (Hoang et al., 2018). 3D printing technologies have been evolving rapidly, and digital light processing (DLP)-based printing is now used to fabricate scaffolds or molds to embed cells in hydrogel directly in a cell culture dish (Ma et al., 2019). Another method is layering hiPSC-CMs into multi-layered cardiac tissue constructs, which successfully recapitulate Torsade de Pointes in vitro (Kawatou et al., 2017). 
Overall, there is a growing effort to learn the appropriate technology and materials to design a reproducible, efficient and affordable 3D culture system, such advancement will be a great step toward the generation of mature, functional cardiomyocytes.

\section{Enhancing hiPSC-CMs Maturation in 3D Culture Systems}

Although 3D cultures of hiPSC-CMs have great potential to make more matured cardiomyocytes, it is insufficient to do so by itself, and a combination of the above-mentioned maturationpromoting methods worked in $2 \mathrm{D}$ must be utilized within a 3D setting, which includes cell-cell interaction (e.g., fibroblasts) (Zhang et al., 2017; King et al., 2019; Valls-Margarit et al., 2019; Yan et al., 2019), hormones (Balistreri et al., 2017; Huang et al., 2020), and electrical and physiological stimulation. Below, we will provide a brief review of the electrical and physiological stimulation applied to $3 \mathrm{D}$ cultures to reach the ultimate goal.

\section{D Cultures With Biophysical Stimuli}

One of the epoch-making studies was done by Nunes et al. (2013). Embedding hiPSC-CMs with collagen gel into PDMS channel to form a wire-like structure and exposing the tissues to electrical stimuli generate functionally more matured cardiac tissue. To further augment electrical conduction, electrically conductive silicon nanowires or carbon nanotubes were incorporated into hiPSC-CMs spheroids to form an electrically conductive environment (Tan et al., 2015; Roshanbinfar et al., 2019) that is later enhanced by the addition of an exogenous electrical stimulation (Richards et al., 2016). The regimens of electrical stimulation were explored by several groups to achieve T-tubule formation and positive force-frequency relationships (Hirt et al., 2014; Godier-Furnémont et al., 2015). More sophisticated stimulation has been explored, and a high-intensity training regimen of electrical current with gradually increasing frequency, from 2 to $6 \mathrm{~Hz}$ within 2 weeks, followed by another week of stimulation at $2 \mathrm{~Hz}$, achieved hiPSC-CMs with adult-like gene expression, well-developed ultrastructure including T-tubule, better calcium handling and contraction force (Ronaldson-Bouchard et al., 2018).

In the mechanical aspect, passive stretch or application of afterload on 3D tissue of hiPSC-CMs (namely, engineered heart muscle or engineered heart tissue) promoted its structural and functional maturation (Abilez et al., 2018; Leonard et al., 2018). A passive stretch is enough to facilitate metabolic switches in hiPSC-CMs (Ulmer et al., 2018). Moderate afterloads are beneficial on cardiomyocyte maturation, while higher afterloads may be detrimental and cause pathological changes (Leonard et al., 2018). The combinations of both electrical and mechanical

\section{REFERENCES}

Abecasis, B., Gomes-Alves, P., Rosa, S., Gouveia, P. J., Ferreira, L., Serra, M., et al. (2019). Unveiling the molecular crosstalk in a human induced pluripotent stem cell-derived cardiac model. Biotechnol. Bioeng. 116, 1245-1252. doi: 10.1002/ bit. 26929

Abilez, O. J., Tzatzalos, E., Yang, H., Zhao, M.-T., Jung, G., Zöllner, A. M., et al. (2018). Passive stretch induces structural and functional maturation of stimuli - either cyclic stretch or static stress - were also explored (Ruan et al., 2016; LaBarge et al., 2019). In such conditions, hiPSC-CMs displayed a more matured signature than a single stimulus.

\section{CONCLUSION AND FUTURE PROSPECTS}

As we summarized, the maturity of hiPSC-CMs is getting better by numerous efforts. However, methods still need further improvement to reach the desired degree of maturity. Other aspects lacked in this field are to determine definitive maturity of hiPSC-CMs rather than relative measurements and some mechanistic insights why cells are maturing with particular stimuli. Transcriptome-based assay followed by bioinformatics would be one way to define the maturity and uncover mechanistic insights (Uosaki et al., 2015), though more transcriptome data of human hearts in late fetus and postnatal periods is required. As we noted, human cardiomyocytes require years to complete their maturation in a human heart (Vreeker et al., 2014). Therefore, a question remains to be elucidated if and how we can generate adult-like mature hiPSC-CMs at an affordable time and cost. In a specific condition in vivo, the maturation process is accelerated (Cho et al., 2017), thus, hopefully, the issue will be resolved in the near future.

\section{AUTHOR CONTRIBUTIONS}

RA summarized the publications for this mini review and drafted the manuscript. RA, TA, and HU wrote the manuscript. RA and NC drew the figures. HU finalized the manuscript.

\section{FUNDING}

This work was supported by Fund for the Promotion of Joint International Research [Fostering Joint International Research (B), 19KK0219] from Japan Society for the Promotion of Science, Takeda Science Foundation, and the Grant for Basic Research of the Japanese Circulation Society to HU.

\section{ACKNOWLEDGMENTS}

We would like to thank all the members in Division of Regenerative Medicine, Center for Molecular Medicine, Jichi Medical University for helpful discussion.

engineered heart muscle as predicted by computational modeling: structural and functional maturation of EHMs. Stem Cells 36, 265-277. doi: 10.1002/stem. 2732

Balistreri, M., Davis, J. A., Campbell, K. F., Da Rocha, A. M., Treadwell, M. C., and Herron, T. J. (2017). Effect of glucose on 3D cardiac microtissues derived from human induced pluripotent stem cells. Pediatr. Cardiol. 38, 1575-1582. doi: 10.1007/s00246-0171698-2 
Bedada, F. B., Chan, S. S.-K., Metzger, S. K., Zhang, L., Zhang, J., Garry, D. J., et al. (2014). Acquisition of a quantitative, stoichiometrically conserved ratiometric marker of maturation status in stem cell-derived cardiac myocytes. Stem Cell Rep. 3, 594-605. doi: 10.1016/j.stemcr.2014.07.012

Bergmann, O., Bhardwaj, R. D., Bernard, S., Zdunek, S., Barnabé-Heider, F., Walsh, S., et al. (2009). Evidence for cardiomyocyte renewal in humans. Science 3, 98-102. doi: 10.1126/science. 1164680

Bers, D. M. (2002). Cardiac excitation-contraction coupling. Nature 10, 198-205.

Bilic, J., and Izpisua Belmonte, J. C. (2012). Concise review: induced pluripotent stem cells versus embryonic stem cells: close enough or yet too far apart? Stem Cells 30, 33-41. doi: 10.1002/stem.700

Branco, M. A., Cotovio, J. P., Rodrigues, C. A. V., Vaz, S. H., Fernandes, T. G., Moreira, L. M., et al. (2019). Transcriptomic analysis of 3D cardiac differentiation of human induced pluripotent stem cells reveals faster cardiomyocyte maturation compared to 2D culture. Sci. Rep. 9:9229. doi: 10. 1038/s41598-019-45047-9

Burridge, P. W., Keller, G., Gold, J. D., and Wu, J. C. (2012). Production of de novo cardiomyocytes: human pluripotent stem cell differentiation and direct reprogramming. Cell Stem Cell 10, 16-28. doi: 10.1016/j.stem.2011. 12.013

Burridge, P. W., Matsa, E., Shukla, P., Lin, Z. C., Churko, J. M., Ebert, A. D., et al. (2014). Chemically defined generation of human cardiomyocytes. Nat. Methods 11, 855-860. doi: 10.1038/nmeth.2999

Cardoso-Moreira, M., Halbert, J., Valloton, D., Velten, B., Chen, C., Shao, Y., et al. (2019). Gene expression across mammalian organ development. Nature 571, 505-509. doi: 10.1038/s41586-019-1338-5

Chan, Y.-C., Ting, S., Lee, Y.-K., Ng, K.-M., Zhang, J., Chen, Z., et al. (2013). Electrical stimulation promotes maturation of cardiomyocytes derived from human embryonic stem cells. J. Cardiovasc. Trans. Res. 6, 989-999. doi: 10.1007/ s12265-013-9510-Z

Chen, R., He, J., Wang, Y., Guo, Y., Zhang, J., Peng, L., et al. (2019). Qualitative transcriptional signatures for evaluating the maturity degree of pluripotent stem cell-derived cardiomyocytes. Stem Cell Res. Ther. 10:113. doi: 10.1186/s13287019-1205-1

Cho, G.-S., Lee, D. I., Tampakakis, E., Murphy, S., Andersen, P., Uosaki, H., et al. (2017). Neonatal transplantation confers maturation of PSC-derived cardiomyocytes conducive to modeling cardiomyopathy. Cell Rep. 18, 571-582. doi: 10.1016/j.celrep.2016.12.040

Chong, J. J. H., Yang, X., Don, C. W., Minami, E., Liu, Y.-W., Weyers, J. J., et al. (2014). Human embryonic-stem-cell-derived cardiomyocytes regenerate non-human primate hearts. Nature 510, 273-277. doi: 10.1038/nature13233

Chun, Y. W., Balikov, D. A., Feaster, T. K., Williams, C. H., Sheng, C. C., Lee, J.-B., et al. (2015). Combinatorial polymer matrices enhance in vitro maturation of human induced pluripotent stem cell-derived cardiomyocytes. Biomaterials 67 , 52-64. doi: 10.1016/j.biomaterials.2015.07.004

Correia, C., Koshkin, A., Duarte, P., Hu, D., Carido, M., Sebastião, M. J., et al. (2018). 3D aggregate culture improves metabolic maturation of human pluripotent stem cell derived cardiomyocytes. Biotechnol. Bioeng. 115, 630-644. doi: 10.1002/bit.26504

Correia, C., Koshkin, A., Duarte, P., Hu, D., Teixeira, A., Domian, I., et al. (2017). Distinct carbon sources affect structural and functional maturation of cardiomyocytes derived from human pluripotent stem cells. Sci. Rep. 7:8590. doi: 10.1038/s41598-017-08713-4

Dattola, E., Parrotta, E. I., Scalise, S., Perozziello, G., Limongi, T., Candeloro, P., et al. (2019). Development of 3D PVA scaffolds for cardiac tissue engineering and cell screening applications. RSC Adv. 9, 4246-4257. doi: 10.1039/ c8ra08187e

DeLaughter, D. M., Bick, A. G., Wakimoto, H., McKean, D., Gorham, J. M., Kathiriya, I. S., et al. (2016). Single-cell resolution of temporal gene expression during heart development. Dev. Cell. 39, 480-490. doi: 10.1016/j.devcel.2016. 10.001

Denning, C., Borgdorff, V., Crutchley, J., Firth, K. S. A., George, V., Kalra, S., et al. (2016). Cardiomyocytes from human pluripotent stem cells: from laboratory curiosity to industrial biomedical platform. Biochim. Biophys. Acta $1863(7 \mathrm{Pt}$ B), 1728-1748. doi: 10.1016/j.bbamcr.2015.10.014

Dhamoon, A. S., and Jalife, J. (2005). The inward rectifier current (IK1) controls cardiac excitability and is involved in arrhythmogenesis. Heart Rhythm 2, 316-324. doi: 10.1016/j.hrthm.2004.11.012
Doss, M. X., Diego, J. M. D., Goodrow, R. J., Wu, Y., Cordeiro, J. M., Nesterenko, V. V., et al. (2012). Maximum diastolic potential of human induced pluripotent stem cell-derived cardiomyocytes depends critically on IKr. PLoS One 5:e40288. doi: 10.1371/journal.pone.0040288

Dunn, K. K., and Palecek, S. P. (2018). Engineering scalable manufacturing of highquality stem cell-derived cardiomyocytes for cardiac tissue repair. Front. Med. 24:110. doi: 10.3389/fmed.2018.00110

Duval, K., Grover, H., Han, L.-H., Mou, Y., Pegoraro, A. F., Fredberg, J., et al. (2017). Modeling physiological events in 2D vs. 3D cell culture. Physiology 32, 266-277. doi: 10.1152/physiol.00036.2016

Elliott, D. A., Braam, S. R., Koutsis, K., Ng, E. S., Jenny, R., Lagerqvist, E. L., et al. (2011). NKX2-5eGFP/w hESCs for isolation of human cardiac progenitors and cardiomyocytes. Nat. Methods 8, 1037-1040. doi: 10.1038/nmeth.1740

Funakoshi, S., Miki, K., Takaki, T., Okubo, C., Hatani, T., Chonabayashi, K., et al. (2016). Enhanced engraftment, proliferation, and therapeutic potential in heart using optimized human iPSC-derived cardiomyocytes. Sci. Rep. 6:19111. doi: 10.1038/srep19111

Godier-Furnémont, A. F. G., Tiburcy, M., Wagner, E., Dewenter, M., Lämmle, S., El-Armouche, A., et al. (2015). Physiologic force-frequency response in engineered heart muscle by electromechanical stimulation. Biomaterials 60 , 82-91. doi: 10.1016/j.biomaterials.2015.03.055

Herron, T. J., Rocha, A. M. D., Campbell, K. F., Ponce-Balbuena, D., Willis, B. C., Guerrero-Serna, G., et al. (2016). Extracellular matrix-mediated maturation of human pluripotent stem cell-derived cardiac monolayer structure and electrophysiological function. Circ. Arrhythm. Electrophysiol. 9:e003638. doi: 10.1161/CIRCEP.113.003638

Hinson, J. T., Chopra, A., Nafissi, N., Polacheck, W. J., Benson, C. C., Swist, S., et al. (2015). Titin mutations in iPS cells define sarcomere insufficiency as a cause of dilated cardiomyopathy. Science 349, 982-986. doi: 10.1126/science.aaa5458

Hirt, M. N., Boeddinghaus, J., Mitchell, A., Schaaf, S., Börnchen, C., Müller, C., et al. (2014). Functional improvement and maturation of rat and human engineered heart tissue by chronic electrical stimulation. J. Mol. Cell Cardiol. 74, 151-161. doi: 10.1016/j.yjmcc.2014.05.009

Hoang, P., Wang, J., Conklin, B. R., Healy, K. E., and Ma, Z. (2018). Generation of spatial-patterned early-developing cardiac organoids using human pluripotent stem cells. Nat. Protoc. 13, 723-737. doi: 10.1038/nprot.2018.006

Hoekstra, M., Mummery, C. L., Wilde, A. A. M., Bezzina, C. R., and Verkerk, A. O. (2012). Induced pluripotent stem cell derived cardiomyocytes as models for cardiac arrhythmias. Front. Physiol. 3:346. doi: 10.3389/fphys.2012.00346

Horikoshi, Y., Yan, Y., Terashvili, M., Wells, C., Horikoshi, H., Fujita, S., et al. (2019). Fatty acid-treated induced pluripotent stem cell-derived human cardiomyocytes exhibit adult cardiomyocyte-like energy metabolism phenotypes. Cells 8:E1095. doi: 10.3390/cells8091095

Huang, C. Y., Peres Moreno Maia-Joca, R., Ong, C. S., Wilson, I., DiSilvestre, D., Tomaselli, G. F., et al. (2020). Enhancement of human iPSC-derived cardiomyocyte maturation by chemical conditioning in a $3 \mathrm{D}$ environment. J. Mol. Cell. Cardiol. 138, 1-11. doi: 10.1016/j.yjmcc.2019.10.001

Huethorst, E., Hortigon, M., Zamora-Rodriguez, V., Reynolds, P. M., Burton, F., Smith, G., et al. (2016). Enhanced human-induced pluripotent stem cell derived cardiomyocyte maturation using a dual microgradient substrate. ACS Biomater Sci. Eng. 2, 2231-2239. doi: 10.1021/acsbiomaterials.6b00426

Jacot, J. G., Martin, J. C., and Hunt, D. L. (2010). Mechanobiology of cardiomyocyte development. J Biomech. 43, 93-98. doi: 10.1016/j.jbiomech.2009.09.014

Jung, G., and Bernstein, D. (2014). hiPSC modeling of inherited cardiomyopathies. Curr. Treat Options Cardiovasc. Med. 16:320. doi: 10.1007/s11936-014-0320-7

Kadota, S., Pabon, L., Reinecke, H., and Murry, C. E. (2017). In vivo maturation of human induced pluripotent stem cell-derived cardiomyocytes in neonatal and adult rat hearts. Stem Cell Rep. 8, 278-289. doi: 10.1016/j.stemcr.2016.10.009

Kamakura, T., Makiyama, T., Sasaki, K., Yoshida, Y., Wuriyanghai, Y., Chen, J., et al. (2013). Ultrastructural maturation of human-induced pluripotent stem cell-derived cardiomyocytes in a long-term culture. Circ. J. 77, 1307-1314. doi: 10.1253/circj.cj-12-0987

Karakikes, I., Ameen, M., Termglinchan, V., and Wu, J. C. (2015). Human induced pluripotent stem cell-derived cardiomyocytes: insights into molecular, cellular, and functional phenotypes. Circ. Res. 117, 80-88. doi: 10.1161/CIRCRESAHA. 117.305365

Kawatou, M., Masumoto, H., Fukushima, H., Morinaga, G., Sakata, R., Ashihara, T., et al. (2017). Modelling Torsade de pointes arrhythmias in vitro in 3D 
human iPS cell-engineered heart tissue. Nat. Commun. 8:1078. doi: 10.1038/ s41467-017-01125-y

Kikuchi, C., Bienengraeber, M., Canfield, S., Koopmeiner, A., Schäfer, R., Bosnjak, Z. J., et al. (2015). Comparison of cardiomyocyte differentiation potential between type 1 diabetic donor- and nondiabetic donor-derived induced pluripotent stem cells. Cell Transplant. 24, 2491-2504. doi: 10.3727/ 096368914 X685762

Kim, C., Wong, J., Wen, J., Wang, S., Wang, C., Spiering, S., et al. (2013). Studying arrhythmogenic right ventricular dysplasia with patient-specific iPSCs. Nature 494, 105-110. doi: 10.1038/nature11799

King, O., Kermani, F., Wang, B., Kit-Anan, W., Fourre, J., Randi, A. M., et al. (2019). Endothelial cell regulation of excitation-contraction coupling in induced pluripotent stem cell derived myocardium. Biophys. J. 116:153a. doi: 10.1016/j.bpj.2018.11.850

Klein, I., and Ojamaa, K. (2001). Thyroid hormone and the cardiovascular system. N. Engl. J. Med. 344, 501-509.

Knollmann, B. C. (2013). Induced pluripotent stem cell-derived cardiomyocytes: boutique science or valuable arrhythmia model? Circ. Res. 112, 969-976. doi: 10.1161/CIRCRESAHA.112.300567

Kreipke, R., Wang, Y., Miklas, J. W., Mathieu, J., and Ruohola-Baker, H. (2016). Metabolic remodeling in early development and cardiomyocyte maturation. Semin. Cell Dev. Biol. 52, 84-92. doi: 10.1016/j.semcdb.2016.02.004

Kroll, K., Chabria, M., Wang, K., Häusermann, F., Schuler, F., and Polonchuk, L. (2017). Electro-mechanical conditioning of human iPSC-derived cardiomyocytes for translational research. Prog. Biophys. Mol. Biol. $130(\mathrm{Pt}$ B), 212-222. doi: 10.1016/j.pbiomolbio.2017.07.003

LaBarge, W., Mattappally, S., Kannappan, R., Fast, V. G., Pretorius, D., Berry, J. L., et al. (2019). Maturation of three-dimensional, hiPSC-derived cardiomyocyte spheroids utilizing cyclic, uniaxial stretch and electrical stimulation. PLoS One 14:e0219442. doi: 10.1371/journal.pone.0219442

Lemoine, M. D., Mannhardt, I., Breckwoldt, K., Prondzynski, M., Flenner, F., Ulmer, B., et al. (2017). Human iPSC-derived cardiomyocytes cultured in $3 \mathrm{D}$ engineered heart tissue show physiological upstroke velocity and sodium current density. Sci. Rep. 7, 1-11. doi: 10.1038/s41598-017-05600-w

Leonard, A., Bertero, A., Powers, J. D., Beussman, K. M., Bhandari, S., Regnier, M., et al. (2018). Afterload promotes maturation of human induced pluripotent stem cell derived cardiomyocytes in engineered heart tissues. J. Mol. Cell Cardiol. 118, 147-158. doi: 10.1016/j.yjmcc.2018.03.016

Lewandowski, J., Rozwadowska, N., Kolanowski, T. J., Malcher, A., Zimna, A., Rugowska, A., et al. (2018). The impact of in vitro cell culture duration on the maturation of human cardiomyocytes derived from induced pluripotent stem cells of myogenic origin. Cell Transplant. 27, 1047-1067. doi: 10.1177/ 0963689718779346

Lopaschuk, G. D., and Jaswal, J. S. (2010). Energy metabolic phenotype of the cardiomyocyte during development, differentiation, and postnatal maturation. J. Cardiovasc. Pharmacol. 56, 130-140. doi: 10.1097/FJC.0b013e3181e74a14

Lopaschuk, G. D., Spafford, M. A., and Marsh, D. R. (1991). Glycolysis is predominant source of myocardial ATP production immediately after birth. Am. J. Physiol. Heart Circulat. Physiol. 261, H1698-H1705.

Lundy, S. D., Zhu, W.-Z., Regnier, M., and Laflamme, M. A. (2013). Structural and functional maturation of cardiomyocytes derived from human pluripotent stem cells. Stem Cells Dev. 22, 1991-2002. doi: 10.1089/scd.2012.0490

Ma, J., Guo, L., Fiene, S. J., Anson, B. D., Thomson, J. A., Kamp, T. J., et al. (2011). High purity human-induced pluripotent stem cell-derived cardiomyocytes: electrophysiological properties of action potentials and ionic currents. Am. J. Physiol. Heart Circ. Physiol. 301, H2006-H2017. doi: 10.1152/ajpheart.00694. 2011

Ma, X., Dewan, S., Liu, J., Tang, M., Miller, K. L., Yu, C., et al. (2019). 3D printed micro-scale force gauge arrays to improve human cardiac tissue maturation and enable high throughput drug testing. Acta Biomater. 95, 319-327. doi: 10.1016/j.actbio.2018.12.026

McCain, M. L., Yuan, H., Pasqualini, F. S., Campbell, P. H., and Parker, K. K. (2014). Matrix elasticity regulates the optimal cardiac myocyte shape for contractility. Am. J. Physiol. Heart Circulat. Physiol. 306, H1525-H1539. doi: 10.1152/ ajpheart.00799.2013

Minami, I., Yamada, K., Otsuji, T. G., Yamamoto, T., Shen, Y., Otsuka, S., et al. (2012). A small molecule that promotes cardiac differentiation of human pluripotent stem cells under defined, cytokine- and xeno-free conditions. Cell Rep. 2, 1448-1460. doi: 10.1016/j.celrep.2012.09.015
Mirbagheri, M., Adibnia, V., Hughes, B. R., Waldman, S. D., Banquy, X., and Hwang, D. K. (2019). Advanced cell culture platforms: a growing quest for emulating natural tissues. Mater Horiz. 6, 45-71. doi: 10.1039/c8mh00803e

Nakano, H., Minami, I., Braas, D., Pappoe, H., Wu, X., Sagadevan, A., et al. (2017). Glucose inhibits cardiac muscle maturation through nucleotide biosynthesis. Yelon D, editor. eLife. 6:e29330. doi: 10.7554/eLife.29330

Nunes, S. S., Miklas, J. W., Liu, J., Aschar-Sobbi, R., Xiao, Y., Zhang, B., et al. (2013). Biowire: a platform for maturation of human pluripotent stem cell-derived cardiomyocytes. Nat. Methods. 10, 781-787.

Ogasawara, T., Okano, S., Ichimura, H., Kadota, S., Tanaka, Y., Minami, I., et al. (2017). Impact of extracellular matrix on engraftment and maturation of pluripotent stem cell-derived cardiomyocytes in a rat myocardial infarct model. Sci. Rep. 7, 1-8. doi: 10.1038/s41598-017-09217-x

Parikh, S. S., Blackwell, D. J., Gomez-Hurtado, N., Frisk, M., Wang, L., Kim, K., et al. (2017). Thyroid and glucocorticoid hormones promote functional T-tubule development in human-induced pluripotent stem cell-derived cardiomyocytes. Circ. Res. 121, 1323-1330. doi: 10.1161/CIRCRESAHA.117. 311920

Pesl, M., Pribyl, J., Caluori, G., Cmiel, V., Acimovic, I., Jelinkova, S., et al. (2017). Phenotypic assays for analyses of pluripotent stem cell-derived cardiomyocytes. J. Mol. Recognit. 30:e2602. doi: 10.1002/jmr.2602

Peters, N. S., Severs, N. J., Rothery, S. M., Lincoln, C., Yacoub, M. H., and Green, C. R. (1994). Spatiotemporal relation between gap junctions and fascia adherens junctions during postnatal development of human ventricular myocardium. Circulation 90, 713-725. doi: 10.1161/01.cir.90.2.713

Puri, M. C., and Nagy, A. (2012). Concise review: embryonic stem cells versus induced pluripotent stem cells: the game is on. Stem Cells 30, 10-14. doi: 10.1002/stem.788

Rana, P., Anson, B., Engle, S., and Will, Y. (2012). Characterization of humaninduced pluripotent stem cell-derived cardiomyocytes: bioenergetics and utilization in safety screening. Toxicol. Sci. 130, 117-131. doi: 10.1093/toxsci/ $\mathrm{kfs} 233$

Ribeiro, A. J. S., Ang, Y.-S., Fu, J.-D., Rivas, R. N., Mohamed, T. M. A., Higgs, G. C., et al. (2015). Contractility of single cardiomyocytes differentiated from pluripotent stem cells depends on physiological shape and substrate stiffness. Proc. Natl. Acad. Sci. U.S.A. 112, 12705-12710. doi: 10.1073/pnas.1508073112

Richards, D. J., Tan, Y., Coyle, R., Li, Y., Xu, R., Yeung, N., et al. (2016). Nanowires and electrical stimulation synergistically improve functions of hiPSC cardiac spheroids. Nano Lett. 16, 4670-4678. doi: 10.1021/acs.nanolett.6b02093

Rog-Zielinska, E. A., Craig, M.-A., Manning, J. R., Richardson, R. V., Gowans, G. J., Dunbar, D. R., et al. (2015). Glucocorticoids promote structural and functional maturation of foetal cardiomyocytes: a role for PGC-1 $\alpha$. Cell Death Differ. 22, 1106-1116. doi: 10.1038/cdd.2014.181

Rog-Zielinska, E. A., Thomson, A., Kenyon, C. J., Brownstein, D. G., Moran, C. M., Szumska, D., et al. (2013). Glucocorticoid receptor is required for foetal heart maturation. Hum. Mol. Genet. 22, 3269-3282. doi: 10.1093/hmg/ddt182

Ronaldson-Bouchard, K., Ma, S. P., Yeager, K., Chen, T., Song, L., Sirabella, D., et al. (2018). Advanced maturation of human cardiac tissue grown from pluripotent stem cells. Nature 556, 239-243. doi: 10.1038/s41586-018-0016-3

Roshanbinfar, K., Mohammadi, Z., Sheikh-Mahdi Mesgar, A., Dehghan, M. M., Oommen, O. P., Hilborn, J., et al. (2019). Carbon nanotube doped pericardial matrix derived electroconductive biohybrid hydrogel for cardiac tissue engineering. Biomater. Sci. 7, 3906-3917. doi: 10.1039/c9bm00434c

Ruan, J.-L., Tulloch, N. L., Razumova, M. V., Saiget, M., Muskheli, V., Pabon, L., et al. (2016). Mechanical stress conditioning and electrical stimulation promote contractility and force maturation of induced pluripotent stem cell-derived human cardiac tissue. Circulation 134, 1557-1567. doi: 10.1161/circulationaha. 114.014998

Scuderi, G. J., and Butcher, J. (2017). Naturally engineered maturation of cardiomyocytes. Front. Cell Dev. Biol. 5:50. doi: 10.3389/fcell.2017.00050

Shiba, Y., Gomibuchi, T., Seto, T., Wada, Y., Ichimura, H., Tanaka, Y., et al. (2016). Allogeneic transplantation of iPS cell-derived cardiomyocytes regenerates primate hearts. Nature 538, 388-391. doi: 10.1038/nature19815

Silbernagel, N., Körner, A., Balitzki, J., Jaggy, M., Bertels, S., Richter, B., et al. (2020). Shaping the heart: structural and functional maturation of iPSCcardiomyocytes in 3D-micro-scaffolds. Biomaterials 227:119551. doi: 10.1016/j. biomaterials.2019.119551

Sinnecker, D., Laugwitz, K.-L., and Moretti, A. (2014). Induced pluripotent stem cell-derived cardiomyocytes for drug development and toxicity 
testing. Pharmacol. Ther. 143, 246-252. doi: 10.1016/j.pharmthera.2014. 03.004

Steinhoff, G., Nesteruk, J., Wolfien, M., Große, J., Ruch, U., Vasudevan, P., et al. (2017). Stem cells and heart disease - brake or accelerator? Adv. Drug Deliv. Rev. 120, 2-24. doi: 10.1016/j.addr.2017.10.007

Takahashi, K., Tanabe, K., Ohnuki, M., Narita, M., Ichisaka, T., Tomoda, K., et al. (2007). Induction of pluripotent stem cells from adult human fibroblasts by defined factors. Cell 131, 861-872. doi: 10.1016/j.cell.2007.11.019

Talman, V., and Kivelä, R. (2018). Cardiomyocyte-endothelial cell interactions in cardiac remodeling and regeneration. Front. Cardiovasc. Med. 5:101. doi: 10.3389/fcrm.2018.00101

Tan, Y., Richards, D., Xu, R., Stewart-Clark, S., Mani, S. K., Borg, T. K., et al. (2015). Silicon nanowire-induced maturation of cardiomyocytes derived from human induced pluripotent stem cells. Nano Lett. 15, 2765-2772. doi: 10.1021/ nl502227a

Thavandiran, N., Nunes, S. S., Xiao, Y., and Radisic, M. (2013). Topological and electrical control of cardiac differentiation and assembly. Stem Cell Res. Ther. 4:14. doi: $10.1186 /$ scrt 162

Tiburcy, M., Hudson, J. E., Balfanz, P., Schlick, S., Meyer, T., Chang Liao, M.-L., et al. (2017). Defined engineered human myocardium with advanced maturation for applications in heart failure modeling and repair. Circulation 135, 1832-1847. doi: 10.1161/CIRCULATIONAHA.116.024145

Tohyama, S., Hattori, F., Sano, M., Hishiki, T., Nagahata, Y., Matsuura, T., et al. (2013). Distinct metabolic flow enables large-scale purification of mouse and human pluripotent stem cell-derived cardiomyocytes. Cell Stem Cell 12, 127 137. doi: 10.1016/j.stem.2012.09.013

Ulmer, B. M., Stoehr, A., Schulze, M. L., Patel, S., Gucek, M., Mannhardt, I., et al. (2018). Contractile work contributes to maturation of energy metabolism in hiPSC-derived cardiomyocytes. Stem Cell Rep. 10, 834-847. doi: 10.1016/j. stemcr.2018.01.039

Uosaki, H., Cahan, P., Lee, D. I., Wang, S., Miyamoto, M., Fernandez, L., et al. (2015). Transcriptional landscape of cardiomyocyte maturation. Cell Rep. 13, 1705-1716. doi: 10.1016/j.celrep.2015.10.032

Uosaki, H., Fukushima, H., Takeuchi, A., Matsuoka, S., Nakatsuji, N., Yamanaka, S., et al. (2011). Efficient and scalable purification of cardiomyocytes from human embryonic and induced pluripotent stem cells by VCAM1 surface expression. Prosper F, editor. PLoS One 18:e23657. doi: 10.1371/journal.pone.0023657

Uosaki, H., and Taguchi, Y. (2016). Comparative gene expression analysis of mouse and human cardiac maturation. Genomics Proteomics Bioinformatics 14, 207-215. doi: 10.1016/j.gpb.2016.04.004

Valls-Margarit, M., Iglesias-García, O., Di Guglielmo, C., Sarlabous, L., Tadevosyan, K., Paoli, R., et al. (2019). Engineered macroscale cardiac constructs elicit human myocardial tissue-like functionality. Stem Cell Rep. 13, 207-220. doi: 10.1016/j.stemcr.2019.05.024

van den Berg, C. W., Okawa, S., Chuva de Sousa Lopes, S. M., van Iperen, L., Passier, R., Braam, S. R., et al. (2015). Transcriptome of human foetal heart compared with cardiomyocytes from pluripotent stem cells. Development 142, 3231-3238. doi: $10.1242 /$ dev. 123810

Veerman, C. C., Mengarelli, I., Lodder, E. M., Kosmidis, G., Bellin, M., Zhang, M., et al. (2017). Switch from fetal to adult SCN5A isoform in human induced pluripotent stem cell-derived cardiomyocytes unmasks the cellular phenotype of a conduction disease-causing mutation. J. Am. Heart Assoc. 6:e005135. doi: 10.1161/JAHA.116.005135

Vreeker, A., van Stuijvenberg, L., Hund, T. J., Mohler, P. J., Nikkels, P. G. J., and van Veen, T. A. B. (2014). Assembly of the cardiac intercalated disk during Preand postnatal development of the human heart. Goumans MJ, editor. PLoS One 9:e94722. doi: 10.1371/journal.pone.0094722
Vuorenpää, H., Penttinen, K., Heinonen, T., Pekkanen-Mattila, M., Sarkanen, J.-R., Ylikomi, T., et al. (2017). Maturation of human pluripotent stem cell derived cardiomyocytes is improved in cardiovascular construct. Cytotechnology 69, 785-800. doi: 10.1007/s10616-017-0088-1

Yan, Y., Bejoy, J., Xia, J., Griffin, K., Guan, J., and Li, Y. (2019). Cell population balance of cardiovascular spheroids derived from human induced pluripotent stem cells. Sci. Rep. 9, 1-12. doi: 10.1038/s41598-018-37686-1

Yanagi, K., Takano, M., Narazaki, G., Uosaki, H., Hoshino, T., Ishii, T., et al. (2007). Hyperpolarization-activated cyclic nucleotide-gated channels and T-Type calcium channels confer automaticity of embryonic stem cell-derived cardiomyocytes. Stem Cells 25, 2712-2719. doi: 10.1634/stemcells.20060388

Yang, C., Al-Aama, J., Stojkovic, M., Keavney, B., Trafford, A., Lako, M., et al. (2015). Concise review: cardiac disease modeling using induced pluripotent stem cells. Stem Cells 33, 2643-2651. doi: 10.1002/stem.2070

Yang, L., Soonpaa, M. H., Adler, E. D., Roepke, T. K., Kattman, S. J., Kennedy, M., et al. (2008). Human cardiovascular progenitor cells develop from a KDR+ embryonic-stem-cell-derived population. Nature 453, 524-528. doi: 10.1038/ nature06894

Yang, X., Pabon, L., and Murry, C. E. (2014a). Engineering adolescence: maturation of human pluripotent stem cell-derived cardiomyocytes. Circ Res. 31, 511-523. doi: 10.1161/circresaha.114.300558

Yang, X., Rodriguez, M., Pabon, L., Fischer, K. A., Reinecke, H., Regnier, M., et al. (2014b). Tri-iodo-l-thyronine promotes the maturation of human cardiomyocytes-derived from induced pluripotent stem cells. J. Mol. Cell Cardiol. 72, 296-304. doi: 10.1016/j.yjmcc.2014.04.005

Yang, X., Rodriguez, M. L., Leonard, A., Sun, L., Fischer, K. A., Wang, Y., et al. (2019). Fatty acids enhance the maturation of cardiomyocytes derived from human pluripotent stem cells. Stem Cell Rep. 13, 657-668. doi: 10.1016/j.stemcr. 2019.08.013

Yin, Z., Ren, J., and Guo, W. (2015). Sarcomeric protein isoform transitions in cardiac muscle: a journey to heart failure. Biochim. Biophys. Acta 1852, 47-52. doi: 10.1016/j.bbadis.2014.11.003

Yoshida, S., Miyagawa, S., Fukushima, S., Kawamura, T., Kashiyama, N., Ohashi, F., et al. (2018). Maturation of human induced pluripotent stem cell-derived cardiomyocytes by soluble factors from human mesenchymal stem cells. Mol. Ther. 26, 2681-2695. doi: 10.1016/j.ymthe.2018.08.012

Zhang, W., Kong, C. W., Tong, M. H., Chooi, W. H., Huang, N., Li, R. A., et al. (2017). Maturation of human embryonic stem cell-derived cardiomyocytes (hESC-CMs) in 3D collagen matrix: effects of niche cell supplementation and mechanical stimulation. Acta Biomater. 49, 204-217. doi: 10.1016/j.actbio.2016. 11.058

Zwi, L., Caspi, O., Arbel, G., Huber, I., Gepstein, A., Park, I.-H., et al. (2009). Cardiomyocyte differentiation of human induced pluripotent stem cells. Circulation 13, 1513-1523. doi: 10.1161/circulationaha.109.86 8885

Conflict of Interest: The authors declare that the research was conducted in the absence of any commercial or financial relationships that could be construed as a potential conflict of interest.

Copyright (C) 2020 Ahmed, Anzai, Chanthra and Uosaki. This is an open-access article distributed under the terms of the Creative Commons Attribution License (CC BY). The use, distribution or reproduction in other forums is permitted, provided the original author(s) and the copyright owner(s) are credited and that the original publication in this journal is cited, in accordance with accepted academic practice. No use, distribution or reproduction is permitted which does not comply with these terms. 